\title{
Análise do perfil medicamentoso de idosos polimedicados no município de Sinop - MT
}

\author{
Analysis of the medication profile of polymedicated elderly in the municipality of Sinop-MT \\ Análisis del perfil farmacológico de ancianos polimedicados en el municipio de Sinop-MT \\ Aline Garcia Fedoce ${ }^{1}$, Mario Mateus Sugizaki ${ }^{1}$, Francine Pazini ${ }^{1 \star}$.
}

\begin{abstract}
RESUMO
Objetivo: Analisar o perfil farmacoterapêutico e fatores associados a saúde de idosos polimedicados atendidos em uma Unidade Básica de Saúde (UBS) e que frequentam um Centro de Convivência do Idoso (CCI) no município de Sinop - MT. Métodos: Estudo quantitativo, com 30 idosos, 15 de cada local citado. Os dados foram obtidos por aplicação de formulários específicos durante 5 meses. Os medicamentos foram classificados segundo o Anatomical Therapeutic and Chemical Classification. Os medicamentos inapropriados (MI) para idosos classificados pelo critério de Beers-Fick. Resultados: Ocorreu maior utilização de medicamentos da classe do sistema cardiovascular (SC) (38,7\%) e do sistema nervoso central (SNC) $(20,7 \%)$ na UBS, enquanto os do CCI utilizaram $39,7 \%$ dos medicamentos para SC e para o trato alimentar e metabolismo (TAM) $(20,4 \%)$. Porém, os idosos da UBS obtiveram maior número de $\mathrm{MI}(14,4 \%)$ comparado ao CCI (12,0\%), com um maior número de possíveis interações medicamentosas (IM) (95) versus (46) do CCl. Conclusão: O perfil farmacoterapêutico diferiu entre os grupos estudados, prevalecendo classes do SC, SNC e TAM. O uso de polifarmácia foi comum, com porcentual de MI de 12, 9\% e 14,4\%, e maior quantidade de IM moderadas. A atenção farmacêutica mostra-se crucial para melhora da farmacoterapia em idosos polimedicados.
\end{abstract}

Palavras-chave: Atenção farmacêutica, Idoso, Polimedicação, Uso de medicamentos.

\begin{abstract}
Objective: To analyze the pharmacotherapeutic profile and factors associated with the health of polymedicated elderly people who attended the Basic Health Unit (BHU) or the Community Elderly Center (CEC) in the municipality of Sinop-MT. Methods: Qualitative study with 30 elderly individuals, 15 from each place. The data were obtained by applying specific forms during 5 months. The drugs were classified according to the Anatomical Therapeutic and Chemical Classification. The inappropriate drugs (ID) for the elderly were classified by the Beers-Fick criteria. Results: There was a greater use of drugs to cardiovascular system (CS) (38.7\%) and central nervous system (CNS) $(20.7 \%)$ at the BHU, while those at the CEC used (39.7\%) of drugs to CS and alimentary tract and metabolism (ATM) (20.4\%). However, the elderly at the BHU obtained a higher number of ID (14.4\%) compared to the CEC (12.09\%), with a greater number of possible drug interactions (DI) (95) versus (46) from the ICC. Conclusion: The pharmacotherapeutic profile differed between the groups, with CS, CNS and, ATM classes prevailing. The use of polypharmacy was common, with an ID percentage of $12,0 \%$ and $14.4 \%$ and a greater amount of moderate DI. Pharmaceutical care is crucial for improving pharmacotherapy in polymedicated elderly.
\end{abstract}

Keywords: Pharmaceutical care, Elderly, Polymedication, Medication use.

\section{RESUMEN}

Objetivo: Analizar el perfil farmacoterapéutico y los factores asociados a salud de los ancianos polimedicados atendidos en una Unidad Básica de Salud (UBS) y que asisten a un Centro de Ancianos (CCI) del municipio de Sinop-MT. Métodos: Estudio cuantitativo, con 30 ancianos, 15 de cada localidad mencionada. Los datos se obtuvieron aplicando formularios específicos durante 5 meses. Los fármacos se clasificaron según la Anatomical Therapeutic and Chemical Classification. Medicamentos inapropiados (MI) para ancianos clasificados según el criterio de Beers-Fick. Resultados: Mayor uso de fármacos para clase del sistema

${ }^{1}$ Universidade Federal do Mato Grosso (UFMT), Sinop - MT. * E-mail: francinepazini@gmail.com 
cardiovascular (SC) (38,7\%) y sistema nervioso central (SNC) $(20,7 \%)$ en la UBS, mientras que los del CCI utilizaron mayoritariamente $39,7 \%$ del SC y para el tubo digestivo y metabolismo (TAM) (20,4\%). Todavía, los ancianos de la UBS obtuvieron mayor número de IM (14,4\%) en comparación con el ICC $(12,0 \%)$, con mayor número de posibles interacciones medicamentosas (IM) (95) frente a (46) del ICC. Conclusion: EI perfil farmacoterapéutico difirió los grupos estudiados, predominando las clases de SC, SNC y TAM. El uso de polifarmacia fue común, con porcentajes de IM con 12, 9\% y 14,4\%, y mayor cantidad de IM moderadas. La atención farmacéutica es fundamental para mejorar la farmacoterapia en ancianos polimedicados.

Palabras clave: Atención farmacéutica, Ancianos, Polimedicación, Uso de medicamentos.

\section{INTRODUÇÃO}

O aumento do perfil demográfico de idosos tornou-se exponencial nas últimas décadas. Fato este relacionado ao aumento da expectativa de vida devido a ações na saúde pública, melhora da qualidade de vida, diminuição da taxa de fecundidade, entre outros (MENESES LL, et al., 2010). Em 2010, os idosos no Brasil contabilizaram cerca de 10,8\%, contudo em 2050 estima-se que este número dobrará representando cerca de $22,71 \%$ da população total (IBGE, 2010). Assim, os idosos são um grupo populacional com grande crescimento e representam os maiores consumidores de medicamentos, uma vez que $90 \%$ dos idosos consomem pelo menos um medicamento e um terço deles cinco ou mais princípios ativos simultaneamente (FERREIRA LS, et al., 2020).

A população idosa demanda maior acesso aos serviços de saúde, em decorrência de fatores como o envelhecimento e a incidência de inúmeras doenças, prevalecendo as doenças crônicas não transmissíveis (DCNT). Eventos que corroboram para uso de polifarmácia (DE OLIVEIRA HSB e CORRADI MLG, 2018).

A polifarmácia é definida como o uso continuado ou simultâneo de 5 ou mais medicamentos por paciente, apresentando etiologia de origem multifatorial, variando desde a presença de DCNT a manifestações clinicas advindas do envelhecimento (SECOLI SR, 2010). Em conjunto, estima-se que 70\% dos idosos brasileiros possuam ao menos uma DCNT, e deste total, $60 \%$ empregam a polifarmácia (OLIVEIRA MVP e BUARQUE DC, 2018).

A hipertensão arterial sistêmica (HAS) é a principal DCNT nos idosos, contribuindo para o maior risco de doenças cardiovasculares que são associadas a alta mortalidade, morbidade e custos a saúde (BASTOSBARBOSA RG et al., 2012). A HAS é a principal doença associada a presença de polifarmácia em pacientes idosos (LUCCHETTI G, et al., 2010).

Embora a polifarmácia seja uma prática comum e muitas vezes inevitável em decorrência dos processos de envelhecimento, deve ser vista com cautela, devido a vulnerabilidade dos idosos ao uso de medicamentos diante de mudanças fisiopatológicas, declínio da capacidade cognitiva, mental e funcional, o que reflete no uso seguro e racional de medicamentos (ALMEIDA NA, et al., 2017). As alterações no organismo em função da idade modificam os processos de farmacocinética e farmacodinâmica dos medicamentos, e a soma desses fatores resulta no comprometimento da farmacoterapia (PEETERS LG, et al., 2019)

O uso contínuo de vários medicamentos pode predispor ao aumento do risco e gravidade de reações adversas ao medicamento (RAM), interações medicamentosas (IM), toxicidade cumulativa, erros de medicação, diminuição da adesão ao tratamento e elevação da morbimortalidade (SECOLI SR, 2010). É destacado um risco de RAM de $8 \%$ quando se utiliza 2 medicamentos, sendo este aumentado para $50 \%$ com o uso de 5 medicamentos e 100\% para quem utiliza 8 medicamentos (CARVALHO FA, et al., 2017). O monitoramento farmacoterapêutico, devido a maior incidência de IM e RAM em decorrência da polifarmácia, é capaz de identificar possíveis IM e medicamentos potencialmente inapropriados para idosos (LOPES LM, et al., 2016).

Diante da necessidade do acompanhamento contínuo do paciente geriátrico e a demanda de medicamentos seguros que se adequem as peculiaridades fisiopatológicas desta população, o profissional farmacêutico é capaz de promover o uso seguro e racional de medicamentos através da atenção farmacêutica (AF) (CORREIA W e TESTON APM, 2020). Os benefícios da AF integrada a equipe multidisciplinar de saúde 
possibilitam a melhora na qualidade de vida e diminuição dos problemas relacionados ao medicamento (PRM) que afetam os idosos. A AF engloba benefícios farmacoterapêuticos a partir de macrocomponentes, incluindo a análise do perfil farmacoterapêutico e posterior acompanhamento clínico (MENESES LL, et al., 2010).

Neste contexto o critério de Beers-Fick pode ser utilizado como uma ferramenta eficaz para identificar medicamentos considerados potencialmente inapropriados, devido a eficácia terapêutica reduzida ou ao maior risco de efeitos adversos em comparação aos benefícios (AMERICAN GERIATRICS SOCIETY, 2012).

O objetivo deste estudo foi analisar o perfil farmacoterapêutico incluindo classes medicamentosas mais utilizadas, potenciais IM, medicamentos inapropriados e fatores associados a saúde de idosos polimedicados, atendidos em uma Unidade Básica de Saúde e de frequentadores de um Centro de Convivência, ambos no município de Sinop - MT.

\section{MÉTODOS}

O presente estudo caracteriza-se como um estudo observacional quantitativo. As entrevistas foram realizadas em uma Unidade Básica de Saúde (UBS) durante reuniões mensais do hiperdia e em um Centro de Convivência do Idoso $(\mathrm{CCl})$ durante atividades semanais, no município de Sinop, MT, durante um período de 5 meses de janeiro a maio de 2016. O acompanhamento foi realizado com 15 idosos de cada grupo, escolhidos por conveniência, totalizando 30 pessoas.

As variáveis dependentes consideradas foram: idade acima de 60 anos, utilização de polifarmácia com 5 ou mais medicamentos e indivíduos portadores de hipertensão arterial sistêmica. Os medicamentos de uso tópico ou fitoterápicos não foram considerados. Os usuários deveriam concordar em participar da pesquisa voluntariamente mediante assinatura em Termo de Consentimento Livre e Esclarecido (TCLE). O trabalho foi realizado através da aplicação de formulários elaborados pela equipe de pesquisa de acordo com formulários baseados em Machuca M, et al. (2003) e Lima RA e Zampieri ALT (2009).

Os medicamentos utilizados pelos usuários foram analisados seguindo a Anatomical Therapeutic and Chemical Classification (ATC) do Nordic Council of Medicines, classificados de acordo com primeiro e segundo nível (WHO, 2020). A utilização de medicamentos potencialmente inadequados para o uso em idosos foi estabelecida de acordo com o critério de Beers-Fick. As análises das possíveis IM foram realizadas através da consulta de base de dados do Micromedex® (HEALTHCARE T, 2008).

A análise temática e estatística dos dados foi realizada mediante leitura do material obtido confrontandoos com o referencial teórico. Este trabalho seguiu os critérios do Conselho Nacional de Saúde que regulamenta a pesquisa envolvendo seres humanos, tendo sido aprovado pelo Comitê de Ética em Pesquisa da Universidade Federal de Mato Grosso sob os pareceres n 405.582 e n 850.782.

\section{RESULTADOS}

Dos 15 idosos entrevistados na UBS selecionada, 12 eram mulheres e 3 homens, com idade entre 60 a 83 anos e idade média de 69,6 (DP $\pm 6,17$ anos). No $\mathrm{CCl}$, dos 15 idosos entrevistados, 10 eram mulheres e 5 homens, a idade média foi de 72,4 (DP $\pm 8,59$ anos) variando de 60 e a 87 anos. Quanto a escolaridade, 5 idosos da UBS eram analfabetos, 8 tinham ensino fundamental incompleto, 1 ensino fundamental completo $\mathrm{e}$ 1 idosa com ensino superior. $\mathrm{No} \mathrm{CCl}, 2$ idosos eram analfabetos e os outros 13 possuíam ensino fundamental incompleto.

Em relação à moradia, todos os idosos atendidos na UBS moravam acompanhados. Os idosos do $\mathrm{CCI}$ apenas 2 dos 15 entrevistados moravam sozinhos. Referente a renda salarial, na UBS, dos 15 entrevistados, 13 possuíam renda inferior a 3 salários, e somente 2 com renda superior a essa margem salarial. No $\mathrm{CCl}$, dos 15 entrevistados, 7 possuíam renda superior a 3 salários mínimos e os outros 6 idosos com rendas inferiores a 3 salários mínimos.

Ao considerar os planos de saúde, os idosos da UBS não possuíam plano de saúde, sendo completamente dependentes da assistência médica e aquisição de medicamentos através do SUS. No CCI, 5 dos 15 
entrevistados possuíam plano de saúde, alegando ter boa cobertura, com utilização de consultas médicas particulares e para aquisição de medicamentos. O restante dos idosos relatou a dependência no SUS para assistência médica e aquisição de medicamentos.

Quanto ao uso de medicamentos classificados de acordo com a ATC, o número total de medicamentos utilizados pelos 15 idosos da UBS foram de 111 medicamentos, distribuídos em 48 apresentações farmacêuticas diferentes, média de 7 medicamentos por paciente (DP $\pm 2,6$ ), com o uso máximo de 15 medicamentos. No CCl o número total de medicamentos utilizados foi de 93 medicamentos, distribuídos em 47 apresentações farmacêuticas diferentes, média de 6 medicamentos por paciente (DP $\pm 1,2$ ) e um número máximo de 8 medicamentos.

Referente à classificação dos subgrupos terapêuticos (segundo a ATC), foi observado na UBS, maior utilização de medicamentos para o sistema cardiovascular (SC) (38,7\%) (Tabelas 1). O segundo grupo de medicamentos mais utilizados foram os medicamentos com ação no SNC $(20,7 \%)$.

Para os idosos do CCI, verificou-se maior porcentagem referente a medicamentos do SC $43(39,7 \%)$, e trato alimentar e metabolismo (TAM) 19 (20,43\%) (Tabela 1).

Tabela 1 - Classificação dos medicamentos utilizados pelos usuários da UBS e do CCI de acordo com ATC.

\begin{tabular}{|c|c|c|c|}
\hline Grupo de estudo & Grupo de medicamentos & no de medicamentos & Porcentagem \\
\hline $\begin{array}{l}\text { UBS } \\
\text { CCI }\end{array}$ & C- Sistema Cardiovascular & $\begin{array}{l}43 \\
37\end{array}$ & $\begin{array}{l}38,7 \% \\
39,7 \%\end{array}$ \\
\hline $\begin{array}{l}\text { UBS } \\
\mathrm{CCl}\end{array}$ & A- Trato Alimentar e Metabolismo & $\begin{array}{l}10 \\
19\end{array}$ & $\begin{array}{r}9,0 \% \\
20,4 \%\end{array}$ \\
\hline $\begin{array}{l}\text { UBS } \\
\text { CCI }\end{array}$ & N- Sistema Nervoso & $\begin{array}{c}23 \\
6\end{array}$ & $\begin{array}{l}20,7 \% \\
6,4 \%\end{array}$ \\
\hline $\begin{array}{l}\text { UBS } \\
\mathrm{CCl}\end{array}$ & M- Sistema Musculo esquelético & $\begin{array}{c}13 \\
7\end{array}$ & $\begin{array}{l}11,7 \% \\
7,5 \%\end{array}$ \\
\hline $\begin{array}{l}\text { UBS } \\
\mathrm{CCl}\end{array}$ & $\begin{array}{l}\text { B- Sangue e órgãos do sistema } \\
\text { hematopoiético }\end{array}$ & $\begin{array}{l}12 \\
12\end{array}$ & $\begin{array}{l}10,8 \% \\
12,9 \%\end{array}$ \\
\hline $\begin{array}{l}\text { UBS } \\
\mathrm{CCl}\end{array}$ & R-Sistema Respiratório & $\begin{array}{l}1 \\
2\end{array}$ & $\begin{array}{l}0,9 \% \\
2,1 \%\end{array}$ \\
\hline $\begin{array}{l}\text { UBS } \\
\mathrm{CCl}\end{array}$ & J- Antibacterianos para uso sistêmico & $\begin{array}{l}4 \\
-\end{array}$ & $\begin{array}{c}3,6 \% \\
-\end{array}$ \\
\hline $\begin{array}{l}\text { UBS } \\
\mathrm{CCl}\end{array}$ & P- Antiparasitários, repelentes e inseticidas & $\begin{array}{l}2 \\
-\end{array}$ & $\begin{array}{c}1,8 \% \\
-\end{array}$ \\
\hline $\begin{array}{l}\text { UBS } \\
\mathrm{CCl}\end{array}$ & S- Órgãos sensoriais & 2 & $1,8 \%$ \\
\hline $\begin{array}{l}\text { UBS } \\
\mathrm{CCl}\end{array}$ & G- Trato geniturinário e hormônios sexuais & $\overline{9}$ & $9.6 \%$ \\
\hline $\begin{array}{l}\text { UBS } \\
\mathrm{CCl}\end{array}$ & $\begin{array}{l}\text { Medicamentos não classificados pelo } \\
\text { código ATC }\end{array}$ & $\begin{array}{l}1 \\
1\end{array}$ & $\begin{array}{l}0,9 \% \\
1,0 \%\end{array}$ \\
\hline & & Total & Total \\
\hline $\begin{array}{l}\text { UBS } \\
\mathrm{CCl}\end{array}$ & & $\begin{array}{c}111 \\
93\end{array}$ & $\begin{array}{l}100 \% \\
100 \%\end{array}$ \\
\hline
\end{tabular}

Fonte: Fedoce AG, et al., 2020.

Nos idosos da UBS os do SC com prevalência de diuréticos (32,5\%) e agentes com ação no sistema renina angiotensina (30,2\%), do SNC com predomínio do uso de analgésicos $(60 \%)$, antidepressivos (20\%), e dos antiepiléticos (10\%) (Tabela 2). Valores semelhantes à UBS foram obtidos com os idosos do $\mathrm{CCl}$, perfazendo em sua maioria agentes com ação no sistema renina angiotensina $(31,4 \%)$ e diuréticos $(28,6 \%)$ do grupo do SC. Os medicamentos para distúrbios ácidos estomacais representaram 47,4\% dos 19 medicamentos usados para TAM (Tabela 2). 
Tabela 2 - Segundo nível de classificação dos medicamentos dos usuários da UBS e do CCI pela ATC.

\begin{tabular}{|c|c|c|c|c|}
\hline $\begin{array}{l}\text { Grupo de } \\
\text { estudo }\end{array}$ & $\begin{array}{l}1^{\circ} \text { nível de } \\
\text { classificação }\end{array}$ & $2^{\circ}$ nível de classificação & $\begin{array}{c}\mathrm{n}^{\circ} \text { de } \\
\text { medicamentos }\end{array}$ & Porcentagem \\
\hline $\begin{array}{l}\text { UBS } \\
\mathrm{CCl}\end{array}$ & \multirow{11}{*}{$\begin{array}{l}\text { C- Sistema } \\
\text { Cardiovascular }\end{array}$} & $\begin{array}{l}\text { C01 Terapia Cardíaca, } \\
\text { vasodilatadores }\end{array}$ & $\begin{array}{l}2 \\
1\end{array}$ & $\begin{array}{l}4.6 \% \\
2,9 \%\end{array}$ \\
\hline $\begin{array}{l}\text { UBS } \\
\mathrm{CCl}\end{array}$ & & C01B Antiarrítmicos & $\overline{2}$ & $\overline{5}, \overline{-} \%$ \\
\hline $\begin{array}{l}\text { UBS } \\
\text { CCI }\end{array}$ & & C03 Diuréticos & $\begin{array}{l}14 \\
10\end{array}$ & $\begin{array}{l}32,5 \% \\
28,6 \%\end{array}$ \\
\hline $\begin{array}{l}\text { UBS } \\
\text { CCI }\end{array}$ & & C05 Vasoprotetores & $\begin{array}{l}1 \\
1\end{array}$ & $\begin{array}{l}2,3 \% \\
2,9 \%\end{array}$ \\
\hline $\begin{array}{l}\text { UBS } \\
\mathrm{CCl}\end{array}$ & & $\begin{array}{l}\text { C08 Bloqueadores seletivos do } \\
\text { canal de cálcio }\end{array}$ & $\begin{array}{l}1 \\
3\end{array}$ & $\begin{array}{l}2,3 \% \\
8,6 \%\end{array}$ \\
\hline $\begin{array}{l}\text { UBS } \\
\text { CCI }\end{array}$ & & C07 Agentes beta bloqueadores & $\begin{array}{l}6 \\
4\end{array}$ & $\begin{array}{l}13,9 \% \\
11,4 \%\end{array}$ \\
\hline $\begin{array}{l}\text { UBS } \\
\text { CCI }\end{array}$ & & $\begin{array}{l}\text { C07AG Agentes alpha e beta } \\
\text { bloqueadores }\end{array}$ & $\begin{array}{l}2 \\
-\end{array}$ & $\begin{array}{c}4,6 \% \\
-\end{array}$ \\
\hline $\begin{array}{l}\text { UBS } \\
\text { CCI }\end{array}$ & & $\begin{array}{l}\text { C07C Agentes beta bloqueadores } \\
\text { em associação com diuréticos }\end{array}$ & $\overline{1}$ & $2, \overline{9} \%$ \\
\hline $\begin{array}{l}\text { UBS } \\
\mathrm{CCl}\end{array}$ & & $\begin{array}{l}\text { C09 Agentes com ação no sistema } \\
\text { renina angiotensina }\end{array}$ & $\begin{array}{l}13 \\
11\end{array}$ & $\begin{array}{l}30,2 \% \\
31,4 \%\end{array}$ \\
\hline $\begin{array}{l}\text { UBS } \\
\mathrm{CCl}\end{array}$ & & $\begin{array}{l}\text { C10 Agentes modificadores de } \\
\text { lipídios }\end{array}$ & $\begin{array}{l}4 \\
2\end{array}$ & $\begin{array}{l}9,3 \% \\
5,7 \%\end{array}$ \\
\hline $\begin{array}{l}\text { UBS } \\
\mathrm{CCl}\end{array}$ & & Total & $\begin{array}{l}37 \\
35\end{array}$ & $\begin{array}{l}100 \% \\
100 \%\end{array}$ \\
\hline \multirow{6}{*}{ UBS } & \multirow{6}{*}{$\begin{array}{l}\text { N-Sistema } \\
\text { Nervoso }\end{array}$} & N02 Analgésicos & 12 & $60,0 \%$ \\
\hline & & N06A Antidepressivos & 4 & $20,0 \%$ \\
\hline & & N07C Antivertigem & 1 & $5,0 \%$ \\
\hline & & N03 Psicolépticos & 2 & $10,0 \%$ \\
\hline & & N05B Ansiolíticos & 1 & $5,0 \%$ \\
\hline & & Total & 20 & $100 \%$ \\
\hline \multirow{6}{*}{$\mathrm{CCl}$} & \multirow{6}{*}{$\begin{array}{l}\text { T- Trato } \\
\text { alimentar e } \\
\text { metabolismo }\end{array}$} & $\begin{array}{l}\text { A02 Fármacos para distúrbios ácidos } \\
\text { estomacais }\end{array}$ & 9 & $47,4 \%$ \\
\hline & & $\begin{array}{l}\text { A03 Drogas usadas em desordens } \\
\text { funcionas gastrointestinais }\end{array}$ & 1 & $5,3 \%$ \\
\hline & & A06 Drogas para Constipação & 1 & $5,3 \%$ \\
\hline & & $\begin{array}{l}\text { A07 Antidiarreico, anti-inflamatório } \\
\text { intestinal/antibacteriano }\end{array}$ & 1 & $5,3 \%$ \\
\hline & & A10 Fármacos usados em diabetes & 7 & $36,8 \%$ \\
\hline & & Total & 19 & $100 \%$ \\
\hline
\end{tabular}

Fonte: Fedoce AG, et al., 2020.

De acordo com critério Beers-Fick, dos 111 medicamentos utilizados por idosos da UBS, 14,4\% foram considerados potencialmente inapropriados considerando repetições. Os mais consumidos foram os inibidores não seletivos da COX, relaxantes musculares e antiespasmódicos, 31,3\% dos 16 medicamentos inapropriados. Em relação aos 93 medicamentos utilizados por idosos no $\mathrm{CCl}, 12$ medicamentos correspondendo a $12,9 \%$ foram considerados inapropriados. A prevalência foi de $16,6 \%$ para o uso de antiarrítmicos, antagonista alfa adrenoreceptor, antidepressivos e estrógenos. 
Ao analisar as possíveis IM da UBS, contabilizaram-se 95 no total, com uma média de 6 possíveis interações por idoso. Das 95 possíveis interações analisadas segundo a classificação referente à gravidade, $83(86,3 \%)$ interações foram classificadas como moderadas, $10(11,6 \%)$ como graves e $2(2,1 \%)$ como leves. Em relação ao embasamento científico das possíveis IM, de um total de 95 interações analisadas, 57 apresentaram bom embasamento científico, 15 classificaram-se como excelente, e 23 apresentaram fraco embasamento científico.

As IM moderadas foram as mais frequentes, perfazendo $86,3 \%$ das 95 interações analisadas. Os medicamentos mais envolvidos nestas IM e suas quantidades observadas foram: ácido acetil salicílico (AAS) $x$ hidroclorotiazida (6), reduz efeito anti-hipertensivo e diurético; AAS $x$ captopril (5), diminui a efetividade do captopril; AAS $x$ diclofenaco sódico (4), risco de sangramento aumentado; e captopril $x$ hidroclorotiazida (4), aumento do risco de hipotensão postural. As interações com AAS $x$ anti-hipertensivo são doses dependentes e o efeito anti-hipertensivo associa-se com a diminuição das prostaglandinas renais vasodilatoras. A associação de dois anti-inflamatórios não esteroidais (AINES) como o AAS e diclofenaco pode aumentar o risco de sangramentos e efeitos adversos gastrointestinais, devido a diminuição da produção de tromboxanos e prostaglandinas gástricas (RANG H, et al., 2015).

No CCI, foram observados 47 potenciais IM, perfazendo uma média de 3,13 possíveis interações por idoso. Dessas 47 possíveis IM, $35(74,5 \%)$ foram consideradas moderadas, $8(17,0 \%)$ graves e $4(8,5 \%)$ leves. Referente ao embasamento científico das possíveis interações, 28 foram classificados com bom embasamento cientifico, 10 excelente e 9 com fraco.

As IM moderadas foram também as mais frequentes, perfazendo $74,5 \%$ do total de 45 das interações analisadas. Os medicamentos mais envolvidos nestas IM e suas quantidades observadas foram: AAS $x$ hidroclorotiazida (5), captopril $x$ hidroclorotiazida (4), AAS x captopril (3); e AAS x atenolol (3), com diminuição do efeito anti-hipertensivo.

\section{DISCUSSÃO}

Referente à escolaridade, estudos mostram que idosos com maior grau de instrução possuem melhor capacidade de percepção dos problemas de saúde incluindo medicamentos. Em contraste, os com menor escolaridade apresentam dificuldades na compreensão e conhecimento dos cuidados de promoção da saúde, fatores estes frequentemente associados com uso excessivo de medicamentos e automedicação (AQUINO GA, et al., 2017).

A polifarmácia em idosos aliada a baixa escolaridade é um fator que contribui para o crescimento de DCNT, devido à baixa adesão ao tratamento ou uso inadequado, nos quais agravam condições de saúde préexistentes e potencializam o consumo de medicamentos (COSTA JVG, et al., 2020). Assim, nossos dados corroboram com as informações acima destacadas, uma vez que os idosos do CCl que possuíam maior nível de escolaridade consumiam menor quantidade de medicamentos em comparação aos da UBS.

Ao considerar o acesso a saúde, no Brasil, mais de 70 milhões de brasileiros dependem exclusivamente do SUS, enquanto outros 47 milhões possuem plano de saúde (ANS, 2020). Dos 47 milhões de usuários de plano de saúde, cerca de $14 \%$ ou 6,6 milhões são idosos, representando apenas $22 \%$ da população idosa brasileira (IESS, 2020).

O quadro econômico do país em constante modificação com as alterações sociais propicia altos preços em planos de saúde, especialmente aqueles com ampla cobertura médico assistencial, fato este que influencia a busca de assistência no setor público (ARRAIS PSD, et al., 2007). Os dados obtidos evidenciam o menor acesso a plano de saúde nos grupos de idosos com menor renda salarial. Pimenta FB, et al. (2015), associam a maior incidência de DCNT, particularmente HAS em idosos com baixo nível socioeconômico e cultural, e que não possuem plano de saúde.

O perfil de idosos de ambos os grupos eram em sua maioria de idosos que moravam acompanhados, apresentando uma vantagem nos cuidados a saúde, visto que os idosos que moram acompanhados apresentam maior aderência aos tratamentos de serviços de saúde, uma vez que os familiares ou cuidadores apresentam maior percepção da saúde do idoso, resultando em uma maior busca por cuidado e acompanhamento médico assistencial (ALMEIDA NA, et al., 2017; SILVA MOM, et al., 2020). 
A média de medicamentos consumida pelos idosos da UBS foi semelhante aos resultados obtidos em outros estudos brasileiros envolvendo a análise do perfil medicamentoso em idosos. A prevalência do consumo de medicamentos foi dos que atuam no SC, seguido de medicamentos para o SNC (DE SANTA HELENA ET, et al., 2018; SILVA RS, et al., 2019).

Em contraste, em outros estudos brasileiros, o perfil medicamentoso foi similar ao obtido pelos idosos do CCI. Os medicamentos mais utilizados foram para o SC, seguido de TAM (SILVEIRA EA, et al., 2014; MUNIZ ECS, et al., 2017).

As classes terapêuticas mais utilizadas estão de acordo com o perfil epidemiológico de DCNTs no Brasil, no qual é observada alta prevalência de fatores de risco cardiovasculares entre os idosos, liderando a causa de morbi-mortalidade nesse público (SILVEIRA EA, et al., 2014).

A prevalência de medicamentos para o SNC relaciona-se ao fato de muitos idosos apresentam insônia, ansiedade e estados de confusão mental, ademais a exclusão social predispõe a quadros depressivos, fatos que podem contribuir para utilização dessa classe medicamentosa (SILVA RS, et al., 2019; MUNIZ ECS, et al., 2017). O perfil do uso de medicamentos encontrado salienta a importância de políticas públicas atentas as necessidades dos processos de envelhecimento para garantir que seu uso seja seguro e eficaz, considerando que os medicamentos para o SNC são os principais responsáveis por RAM (PEREIRA KG, et al., 2017).

Acredita-se que ao implantar programas que possibilitem a manutenção da capacidade cognitiva e funcional do idoso, é possível melhorar a autoestima e qualidade de vida. Os idosos com estratégias de enfrentamento positivas aos aspectos da idade avançada são os que apresentam acesso a níveis de afetos positivos (SOARES E, et al., 2012).

Ademais, a prevenção e tratamento de DCNT e incapacidades funcionais do envelhecimento dependem intimamente do estilo de vida que idosos apresentam, a inadequação desta conduta resulta em baixas condições de saúde e qualidade de vida (JOBIM FARC e JOBIM EAC, 2015). A exemplo, um estilo de vida menos ativo, com menor prática de exercícios físicos, atividades recreativas e cognitivas, predispõe ao aumento das perdas cognitivas, afetando diretamente o bem-estar físico e mental de idosos com DCNT (VARGAS LS, et al., 2014).

Os Centros de Convivência de Idosos buscam promover e desenvolver atividades que promovam melhora na qualidade de vida do idoso, e consequentemente, uma menor predisposição para polifarmacoterapia (MARQUES ADB, et al., 2015). Ao considerar a inserção dos idosos em atividades recreativas desempenhadas no $\mathrm{CCI}$ do presente estudo, evidenciou-se uma menor utilização de medicamentos, o que pode impactar positivamente na melhora da qualidade de vida.

Os medicamentos para o trato alimentar e digestivo, como observado em alta prevalência no CCI, associam-se muitas vezes com sintomas causados na mucosa gástrica devido ao uso excessivo de medicamentos, em consequência da polimedicação (FLORES VB e BENVEGNÚ LA, 2008). Bueno CS, et al. (2012) e Gautério-Abreu DP, et al. (2016) mostram resultados semelhantes aos obtidos no estudo em relação ao uso de medicamentos considerados inapropriados para idosos.

As potenciais IM corroboram com estudo publicado por Gotardelo DR, et al. (2014), que destacou 5,6\% de possíveis IM leves, $81,6 \%$ de moderadas e $12,8 \%$ de maior gravidade. O estudo relata que as IM são mais frequentes em pessoas com idade avançada, do sexo feminino, casados ou morando com outra pessoa, e renda familiar menor ou igual a 3 salários mínimos, com maior ocorrência em portadores de doenças crônicas. Observações similares foram também destacadas por Silveira EA, et al. (2014), que demonstrou prevalência de polimedicação em idosos do sexo feminino, sendo esta 1,76 vezes maior quando comparado ao sexo masculino.

O perfil de usuários de polifarmácia é predominantemente feminino. Este fato pode ser decorrente de que as mulheres apresentam maior expectativa de vida comparada ao homem, maior procura ao atendimento à saúde e programas de saúde, responsáveis em sua maioria pelos cuidados à família, familiarizando-se com os medicamentos (PEREIRA KG, et al., 2017). Neste estudo, em ambos os grupos o sexo feminino foi predominante. 
A utilização da polifarmácia pode resultar na alta incidência de RAM e IM. Entretanto, o uso concomitante de vários medicamentos se faz necessário na maioria dos casos e auxiliam no aumento da expectativa de vida. Sendo assim, a polifarmácia aliada a irracionalidade de uso resulta nos potenciais riscos à saúde do idoso, ao passo que aliada a promoção do uso seguro e racional de medicamentos promove benefícios a saúde do idoso. Salienta-se a importância da AF para promoção dos benefícios associados a utilização da polifarmácia nesta população em especifico (ALMEIDA NA, et al., 2017).

Ambos os grupos de idosos analisados faziam utilização da polimedicação em adição ao uso de medicamentos inapropriados, tornando-os mais susceptíveis a morbidade, mortalidade e uso dos serviços de saúde. Desta forma, cuidados individualizados voltados a AF ao idoso são exigidos, ao considerar múltiplas doenças em associação com alterações na farmacocinética e farmacodinâmica dos medicamentos em uso (BUENO CS, et al., 2012). Vale destacar que o acompanhamento farmacoterapêutico aliado a promoção em saúde, promovidos por uma equipe multidisciplinar propicia aos idosos em situação de vulnerabilidade, o conhecimento e consciência, a respeito do estado de saúde frente aos processos de envelhecimento (DOS SANTOS et al., 2020).

\section{CONCLUSÃO}

Ao analisar o perfil farmacoterapêutico de idosos polimedicados, foi possível concluir que o perfil de medicamentos, medicamentos impróprios e IM difere entre os dois grupos do estudo, agravando-se em mulheres, idosos com menor escolaridade, menor renda salarial e sem acesso a planos de saúde. O perfil medicamentoso observado acompanha demais estudos brasileiros, prevalecendo classes do SC, SNC e TAM. O uso de polifarmácia foi comum entre os idosos analisados, tendo sido observado um porcentual de medicamentos inapropriados de 12, $9 \%$ e 14,4\%, e maior quantidade de IM moderadas. Desta forma, a AF é crucial para a identificação do perfil farmacoterapêutico de idosos polimedicados possibilitando adequações e planejamento da farmacoterapia, no que concerne ao bem-estar físico e mental referente aos processos de envelhecimento.

\section{AGRADECIMENTOS}

Agradecemos a participação dos idosos dos locais do estudo por toda a disponibilidade.

\section{REFERÊNCIAS}

1. AGÊNCIA NACIONAL DE SAÚDE SUPLEMENTAR-ANS, 2020. Disponível em: https://www.ans.gov.br/perfil-dosetor/dados-gerais. Acesso em: 20 de dez de 2020.

2. ALMEIDA NA, et al. Prevalence of and factors associated with polypharmacy among elderly persons resident in the community. Revista Brasileira de Geriatria e Gerontologia, 2017; 20(1): 138-148.

3. AMERICAN GERIATRICS SOCIETY. Beers Criteria Update Expert Panel. American Geriatrics Society updated Beers Criteria for potentially inappropriate medication use in older adults. Journal of American Geriatric Society. 2012; 60: 616-631.

4. AQUINO GA, et al. Factors associated with adherence to pharmacological treatment among elderly persons using antihypertensive drugs. Revista Brasileira de Geriatria e Gerontologia, 2017; 20(1): 111-122.

5. ARRAIS PSD, et al. Aspectos dos processos de prescrição e dispensação de medicamentos na percepção do paciente: estudo de base populacional em Fortaleza, Ceará, Brasil. Cadernos de Saúde Pública, 2007; 23: 927-937.

6. BASTOS-BARBOSA RG, et al. Adesão ao tratamento e controle da pressão arterial em idosos com hipertensão. Arquivos Brasileiros de Cardiologia, 2012; 99(1): 636-641.

7. BUENO CS, et al. Perfil de uso de medicamentos por idosos assistidos pelo Programa de Atenção ao Idoso (PAI) da UNIJUÍ. Revista Brasileira de Geriatria e Gerontologia, 2012; 15(1): 51-61.

8. CARVALHO FA, et al. Riscos da interação medicamentosa em pacientes hipertensos: um estudo em grupo específico de pacientes que fazem uso de antihipertensivos. Journal Health Science Institute, 2017; 35(3): 215- 218.

9. CORREIA W, TESTON APM. Aspectos relacionados à polifarmácia em idosos: um estudo de revisão. Brazilian Journal of Development, 2020; 6(11): 93454-93469.

10. COSTA JVG, et al. Perfil do uso de medicamentos por idosos: sob o olhar farmacêutico. Revista de Psicologia, 2020; 14(52): 158-166.

11. DE OLIVEIRA HSB, CORRADI MLG. Aspectos farmacológicos do idoso: uma revisão integrativa de literatura. Revista de Medicina, 2018; 97(2): 165-176. 
12. DE SANTA HELENA ET, et al. Polifarmácia e padrão de utilização de medicamentos em Pomerode, SC. Arquivos Catarinenses de Medicina, 2018; 47(2): 124-136.

13. DOS SANTOS JMS, et al. Ações educativas em instituições de longa permanência para idosos: uma revisão narrativa da literatura. Brazilian Journal of Development, (2020); 6(3), 12140-12152.

14. FERREIRA LS, et al. Automedicação: prática comum por idosos de um município do norte do Paraná. Brazilian Journal of Development, 2020; 6 (4): 22404-22413.

15. FLORES VB, BENVEGNÚ LA. Perfil de utilização de medicamentos em idosos da zona urbana de Santa Rosa, Rio Grande do Sul, Brasil. Cadernos de Saúde Pública, 2008; 24: 1439-1446.

16. GAUTÉRIO-ABREU DP, et al. Uso de medicamentos inapropriados por pessoas idosas residentes em instituição de longa permanência. Journal of Nursing UFPE, 2016; 10(2): 608-614.

17. GOTARDELO DR, et al. Prevalência e fatores associados a potenciais interações medicamentosas entre idosos em um estudo de base populacional. Revista Brasileira de Medicina de Família e Comunidade, 2014; 9(31): $111-118$.

18. HEALTHCARE, T. In: MICROMEDEX® Healthcare Series, 2008. Disponível em: www.micromedex.com. Acesso em: 20 de ago de 2020.

19. IBGE. INSTITUTO BRASILEIRO DE GEOGRAFIA E ESTATÍSTICA, In: CENSO 2010. Disponível em: www.ibge.gov.br. Acesso em: 20 de ago de 2019.

20. IESS. INSTITUTO DE ESTUDOS DE SAÚDE SUPLEMENTAR, In: PANORAMA dos idosos beneficiários de planos de saúde no Brasil, 2020. Disponível em: https://www.iess.org.br/cms/rep/panorama_dos_idosos.pdf. Acesso em: 20 de dez de 2020.

21. JOBIM FARC, JOBIM EAC. Atividade física, nutrição e estilo de vida no envelhecimento. UNOPAR Cientiefíca, Ciências biológicas e saúde, 2015; 17(4).

22. LIMA RA, ZAMPIERI ALT. Proposta para implementação da atenção farmacêutica na Farmácia Escola de uma Instituição de Ensino Superior (IES) de Anápolis. Monografia (Graduação em Farmácia). Associação Educativa Evangélica-UniEvangélica, Anápolis, 2009.

23. LOPES LM, et al. Use of potentially inappropriate medications by the elderly at home. Ciência \& Saúde Coletiva; 2016; 21: 3429-3438.

24. LUCCHETTI G, et al. Fatores associados à polifarmácia em idosos institucionalizados. Revista Brasileira de Geriatria e Gerontologia, 2010; 13(1): 51-58.

25. MACHUCA M, et al. Método Dáder: manual de acompanhamento farmacoterapêutico. Granada: GIAF-UGR, 2003; 189-194.

26. MARQUES ADB, et al. A vivência da sexualidade de idosos em um centro de convivência. Revista de Enfermagem do Centro-Oeste Mineiro, 2015, 5(3): 1768-1783.

27. MENESES LL, et al. Atenção farmacêutica ao idoso: fundamentos e propostas. Geriatrics, Gerontology and Aging, 2010; 4(3): 154-161.

28. MUNIZ ECS, et al. Análise do uso de medicamentos por idosos usuários de plano de saúde suplementar. Revista Brasileira de Geriatria e Gerontologia, 2017; 20(3): 374-386.

29. OLIVEIRA MVP, BUARQUE DC. Polifarmácia e medicamentos potencialmente inapropriados em idosos admitidos em um hospital terciário. Geriatric Gerontology and Aging, 2018; 12(1): 38-44.

30. PEETERS LEJ, et al. Pharmacokinetic and pharmacodynamic considerations in the treatment of the elderly patient with hypertension. Expert Opinion on Drug Metabolism \& Toxicology, 2019; 15(4): 287-290.

31. PEREIRA KG, et al. Polifarmácia em idosos: um estudo de base populacional. Revista Brasileira de Epidemiologia, 2017; 20: 335-344.

32. PIMENTA FB, et al. Fatores associados a doenças crônicas em idosos atendidos pela Estratégia de Saúde da Família. Ciência \& Saúde Coletiva, 2015; 20: 2489-2498.

33. RANG H, et al. Rang \& Dale Farmacologia. $8^{\text {th }} . e d$. Elsevier. São Paulo, São Paulo; 2015, 798p.

34. SECOLI SR. Polifarmácia: interações e reações adversas no uso de medicamentos por idosos. Revista Brasileira de Enfermagem, 2010; 63(1):136-140.

35. SILVA MOM, et al. Acompanhamento farmacêutico: adesão e problemas relacionados à farmacoterapia de idosos. Journal of Biology \& Pharmacy and Agricultural Management, 2020; 17(2): 399-415.

36. SILVA RS, et al. Condições de saúde de idosos institucionalizados: contribuições para ação interdisciplinar e promotora de saúde. Cadernos Brasileiros de Terapia Ocupacional, 2019; 27(2): 345-356.

37. SILVEIRA EA, et al. Polifarmácia, doenças crônicas e marcadores nutricionais em idosos. Revista Brasileira de Epidemiologia, 2014; 17: 818-829.

38. SOARES E, et al. Capacidade funcional, declínio cognitivo e depressão em idosos institucionalizados: possibilidade de relações e correlações. Revista Temática Kairós Gerontologia, 2012; 15(5): 117-139.

39. VARGAS LS, et al. Influência da diabetes e a prática de exercício físico e atividades cognitivas e recreativas sobre a função cognitiva e emotividade em grupos de terceira idade. Revista Brasileira de Geriatria e Gerontologia, 2014; 17(4): 867-878.

40. WHO. WORLD HEALTH ORGANIZATION. 2020. In: Collaborating centre for drug statistics methodology; Guidelines for ATC classification and DDD assignment. Disponível https://www.whocc.no/atc_ddd_index_and_guidelines/guidelines/. Acesso em: 20 de dez de 2020. 